\title{
On the Prospects For a Unified Social Science: Economics and Sociology
}

\section{Peter Abell}

London School of Economics and Political Science.

Interdisciplinary Institute of Management

Houghton Street, London WC2A 2AE, UK.

p.abell@1se.ac.uk

\begin{abstract}
This paper argues for a closer association between economics and sociology. The latter could benefit from the intellectual rigour of the former. Building a unified socio-economics requires an intellectual rapprochement between the sociologist conception of social interaction and a relaxed version of rational choice. Evolutionary game theory may provide one way forward.
\end{abstract}

Key words: unified social science, economics, sociology.

Resumen. Perspectivas para una ciencia social unificada: economía y sociología

Este artículo argumenta a favor de una colaboración más estrecha entre la economía y la sociología. Esta última podría beneficiarse del rigor intelectual de la primera. La construcción de una socio-economía unificada requiere de una aproximación intelectual entre la concepción sociológica de la interacción social y una versión suavizada de la elección racional. La teoría de juegos evolucionaria puede proporcionar una camino prometedor en ese sentido.

Palabras clave: ciencia social unificada, economía, sociología.

\section{Contents}

1. Introduction

2. Models of the individual

3. Social interaction

4. Combining rational choice and interaction
5. A note on the macro level (level 4) -emergence?

6. Conclusions

References 


\section{Introduction}

There are two rather compelling reasons for posing the question as to whether a unified social science is a realizable possibility. First, and most obviously, the query itself is intellectually intriguing — can we cogently conceive of such a 'science' ${ }^{1}$ and, if so, what would be involved in laying out its foundational precepts? Secondly, and less obviously, though of equal importance, there is a politico-intellectual reason. The standards of rigour and sustained reasoning being so variable across the social sciences, one might hope that any attempt to open up the debate surrounding the issues of unification may have the effect of driving them up. Unacceptable standards are currently widespread and maintained, first, because some disciplines are effectively insulated in the universities behind departmental, disciplinary boundaries and, secondly, because those working to higher standards, whilst being entirely aware of this situation, tend merely to shrug their shoulders with an air of resignation. This seems to me to be morally unacceptable, so one of my objectives in writing this paper is to stimulate a cross-disciplinary debate, which may in turn foster the spread of better practice. I trust this may be a realizable possibility even if unification proves to be but a mirage.

We should start by asking which of the current array of disciplines might be candidates for unification. I shall restrict my attention to economics and sociology and, in a very much more limited way, to psychology. This restriction reflects my own limitations. I write as a sociologist with a lamentably amateur knowledge of economics and psychology but I suspect what I have to say may well have wider implications. There is an obvious sense in which my title is over-ambitious as I shall not reach for unification across the full range of the social sciences. I prefer, however, to remain with the ambitious title in the hope that others, more competent than I, may join the debate - perhaps in the pages of this exciting journal.

A good way to start is by clarifying the intellectual role which the word 'social' might be allowed to perform in our endeavours. A simple diagram which I have found useful in this respect is depicted in Figure $1^{2}$. It points to four types of explanatory questions, each of which at least one of the established social sciences addresses, and all of which when taken together provide a focus for unification. I shall use the word 'social' to indicate that we are usually concerned with explaining a (macro) state of the (social) system (i.e. at the top right-hand corner of the diagram). That is to say, it is not, in general, an ambition of the social sciences to explain per se individual states/actions (i.e. the bottom right-hand

1. I shall use the term 'science' although I know in some quarters it proves controversial. I would be entirely happy for the word to be replaced by a less controversial one. Nothing in what follows entails a commitment to continuity with natural science.

2. The diagram is sometimes called the Coleman Diagram after Coleman (1990). It should be clear that it could be elaborated (a) so that more than two 'levels' are involved (e.g. individual, group, organization, industry, society) and (b) forwards and backwards in time. See Abell (2001). I drop this complexity for the purpose of this paper. 


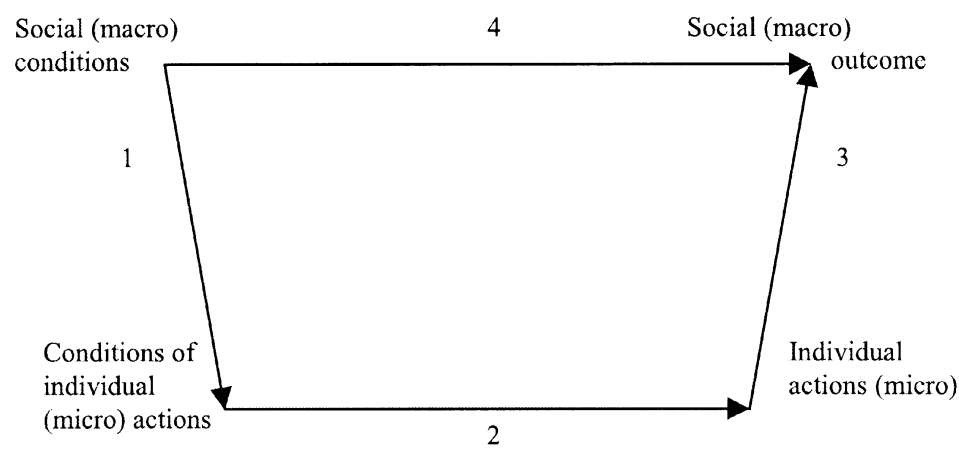

Figure 1.

corner of Figure 1). Such explanations are only sought in so far as they are permitted to contribute to explanations of macro states ${ }^{3}$. Examples could be an (equilibrium) price in competitive markets (from economics) or levels of group cohesion or collective social capital (from sociology).

The arrows in the diagram can stand for a number of types of connection or explanation (causal, aggregation, definitional or even emergence in the case of arrow 3), but for the moment it will expedite the argument to think solely in terms of causal explanations. Consequently, the diagram invites us to speculate about the interplay of four types of causal mechanism. In general, we may conceive of dynamical systems (which may or may not be tending to a steady state) at two different levels of abstraction (see footnote 3 ), the macro and the micro, and pose the question as to their interrelationship. Sociologists will recognize type 4 explanations as essentially Durkheimian; here a prior state of the (social) system causes (ceteris paribus) a later state of the system. Thus, following Durkheim, exogenously established societal norms cause (ceteris paribus) societal rates of suicide. Durkheim argued that such (causal) mechanisms should be conceived as operating sui generis, at the societal level and, as a consequence, appropriately analysed independently of mechanisms 1,2 and $3^{4}$. An economic example of a type 4 mechanism might

3. Sociologists would perhaps also use the word 'social' to emphasize that level 2 explanations (Figure 1) concern the social interaction of at least two actors (see below). Despite my emphasis on explanations of macro states many sociologists often seem unsure as to whether the bottom or top right-hand corner of Figure 1 is the source of explanatory attention. This may be because explanations of type 3 are sometimes no more than a summary or aggregate measure of the distribution of actions/states at the micro level (e.g. the average voting intention). Again, I shall return to these matters.

4. Durkheim, we are told, regarded this approach (i.e. analysis without micro reduction) as justifying sociology as an independent discipline. I am told that Durkheim had a much more complex viewpoint. 
be the level of competition in a market causing the reduction in (equilibrium) price. Economists are, however, much less prone than sociologists to analyse level 4 relationships as sui generis. Nevertheless, a debate continues about whether and, if so, how macro economics should be securely derived from micro foundations. I will return to the nature of this quest but we may note, in passing, that it suggests an encounter with the other three types of mechanism depicted in Figure 1, though, in fact, type 1 explanations are rarely sought by economists 5 .

In general, however, for those social scientists who do not care to adopt a strict Durkheimian perspective, there is an obvious sense in which they will be inclined to see type 4 explanations as, in some manner, derivative of the conjunction of types 1, 2 and 3. So, for example, to take a non-Durkheimian interpretation of suicide rates (top right-hand corner of Figure 1) one would search for types of mechanisms whereby (exogenous) societal norms ${ }^{6}$ impact (type 1) the conditions of individual interactions, then (type 2) where the conditions impact the individual propensity to suicide and, finally, (type 3) where the propensities aggregate ${ }^{7}$ to societal rates of suicide. This simple depiction glosses over a number of conceptual and philosophical issues but prompts the first of a number of major questions which must be addressed if we are to aspire to a unified social science: what sort of model of the individual is (at the micro level 2) appropriate when an explanation of the system state is sought? This observation enables one to draw some conclusions about the limited role which individual psychology will play in any unified social science. Psychologists and, indeed, many sociologists often allege that economists adopt an over-simple model of the individual (i.e. usually rational, calculating and self-interested). Maybe they do, but the important point is, nevertheless, that the social sciences should only adopt the simplest model of the individual consisten $\mathrm{t}^{8}$ with validated psychology theory, which can in turn contribute to an account of the system state. This being the case, the social sciences will not always, or even usually, shift with changing fashions in our understanding of individual psychology. Unfortunately many sociolo-

5. In general economists will take the bottom left-hand corner in Figure 1 as exogenous, comprising preferences and beliefs (expectations), though recently an interest in endogenous preferences and expectations has arisen. This apart, economists tend to model societal (macro) influences on individuals in terms of opportunities.

6. As we shall see below, what should and should not be taken as exogenous is an issue that currently divides the social sciences. Sociologists, because their discipline lacks rigour, tend to criticize economists for taking things as exogenous without themselves facing the technical responsibilities of not doing so. Figure 1 could conceivably invite an endogenous theory of 'social norms' by elaborating the diagram backwards in time (see also footnote 2).

7. Note the type 3 arrow appears here to be one of aggregation (not causality)!

8. The idea here is that the social scientist's model of the individual can ignore minute psychological detail, but the degree to which this is analytically appropriate often depends upon the number of actors at the micro level. It is a matter of what is sometimes called supervenience. Generally the more refined the macro dynamics, the more refined the model of the individual. 
gists have not taken this lesson to heart, with the result that a type of literature has evolved which tries to locate ever more refined ways of understanding individuals and their interactions. Social scientists have very little to learn from this literature?

Sociologists frequently promote their discipline as one which involves the study of social interaction and exchange (Coleman, 1990). Accordingly, level 2 mechanisms then need to capture the idea whereby 'the conditions of action' (bottom left-hand corner of Figure 1) describe how others impact the actions of each focal individual ${ }^{10}$. Instructively, economists are also concerned with economic exchange between (exogenously endowed ${ }^{11}$ ) individuals, but abstract away from detailed descriptions of such exchanges in their most vaunted achievement: general equilibrium theory ${ }^{12}$. They do so to avoid the need to chart the details of non-equilibrium exchanges. How and why they do this is an issue I shall return to, but again it is tied up with the adoption of an appropriate simplification in the search for, in this case, a highly stylized equilibrium macro state.

As soon as the idea of (social) interaction is given a central role in our endeavours, it is essential to draw a basic distinction between those models which are parametric and those which are strategic. If actors can be modelled as responsive to their 'conditions of action', which may, amongst other things, include other actors' actions that may be treated as fixed and are not themselves responsive to the focal actor, then the conditions are parametric. The canonical model here is the aforementioned general equilibrium theory, where at equilibrium fixed prices determine purchasing actions.If, however, we need to understand how actors reason about the way other actors will act in response to their own actions (and vice versa), then the situation becomes strategic. This distinction is central to modern economic theory but rarely explicitly acknowledged by sociologists ${ }^{13}$. It should be, though, and I believe an understanding of how, why and when we should adopt one approach rather than the other may provide one useful ingredient in establishing a unified social science. Indeed, matters go even deeper: sometimes we may wish to model individuals in terms of what, in the natural sciences, is termed an 'independent individual approximation'. That is to say, in order to approximate highly complex micro dynamics one discards the idea of interaction altogether, replacing it with some general (or average) situated environment. Let me take an example from my own research into generalized reciprocity and social capital. A collection of $n$ indi-

9. The exception here might be when the number of actors at level 2 is small.

10. This would be true even if the appropriate micro level were 'above' the individual (e.g.groups in relation to society).

11. Usually endowed with preferences and resources. That is, the bottom left-hand corner of Figure 1 is exogenous: no type 1 mechanisms.

12. I hasten to add that this avoidance of the detail of exchanges is not found in many economic theories, especially game-theoretic strategic theories.

13. One might suggest that sociologists' conception of social interaction is nearly always implicitly strategic (Abell,1996b). 
viduals may be described in terms of the dynamics of 'who helps whom' (a complex network of dyadic strategic interactions ${ }^{14}$ ). So, individual helping actions are 'caused' by specific others helping actions. In practice, however, we may (particularly when $n$ is large) discard the strategic details of the micro dynamics and adopt the standpoint of 'the characteristic individual' in a 'mean helping environment'. It should be borne in mind, nevertheless, that the theoretical generation of such a parametric environment, which provides a picture of the mean effects of other actors on each focal actor in turn, although having the appearance of an exogenous parametric force, impacting each individual, is in fact endogenously generated ${ }^{15}$. Theoretical sociologists have often appeared lax in not appropriately marking this distinction and, in particular, have shown little sensitivity to how the nature of theories may change as $n$ dwindles. Indeed, the literature on agency and structure (e.g. Giddens, 1989) seems to have evolved in complete innocence of these sorts of issues, contributing to what I have elsewhere termed a fugitive paradigm (Abell and Reyniers, 2001). If social theory is to acquire greater intellectual bite, then its practioners will need to transcend this sort of innocence. A drive towards unified theory may, perhaps, help in this respect.

Economists also handle situations of interdependent strategic complexity, inherent in economic exchanges, in a rather similar manner in their general equilibrium theory. Arrow (1983) writes of general equilibrium as follows: 'the key points in the definition are the parametric role of the prices for each individual and the identity of price for all individuals'. Again these prices are generated endogenously but they appear (at equilibrium) rather like exogenous parametric forces. General equilibrium, however, provides no picture as to how equilibrium prices arise. The theoretical structure abstracts away from pre-equilibrium exchange (i.e. interactions) between individuals ${ }^{16}$. So, in both sociology and economics it does appear that, at least when $n$ is large, there may be some common ground in reducing the complexity of strategic interactions to more tractable parametric models. We need to look at this possibility more closely in the context of unification.

14. Generalized reciprocity of help is usually modelled strategically as a trust or Prisoner's Dilemma game. See Abell and Reyniers (2000).

15. Sociologists refer to the use of mean effects of this sort as cross-level or structural effects. In the context of Figure 1, complex (strategic) interactions at level 2 are being approximated by a mean in the appropriate population. One could conceive the mean level concept at the top left corner.

16. Tatonnement is, of course, the word used, under the auspices of the Walrasian auctioneer, to describe the groping towards equilibrium (but this is not based upon pairwise exchanges or interactions). Interestingly, models of dyadic economic exchange of the Edgeworth sort do converge upon competitive equilibrium prices as $n$ increases. Once again we may note that the style of theory which is appropriate depends upon $n$. The convergence of these two ways of looking at markets is a conspicuous sign of the maturity and rigour of economics. Such convergences are common in the natural sciences but totally absent from sociology (I suspect because of the low standards of intellectual rigour in theoretical sociology). 
What apparently most divides the social sciences — particularly economics from sociology - is the concept of equilibrium ${ }^{17}$. Economists will almost invariably start by trying to show how optimizing individuals will drive the system towards a steady state. Sociologists rarely think in these terms. But should they? Should the social sciences be preoccupied with explaining time invariant system states at the top right-hand corner of Figure 1? Sociologists have failed to formulate any general theoretical framework which deploys a clear concept of equilibrium, though much empirical research is conducted and reported in a way which implicitly assumes that distributions (e.g. the distribution of individual states/actors at the bottom right-hand corner of Figure 1) have found a steady state $^{18}$. In fact, this observation raises a further issue concerning explanations at level 3. Macro stability (equilibrium) does not invariably imply micro stability but the reverse may often be true ${ }^{19}$. Neither sociologists nor economists have fully embraced the implications of this fact and thought through the interplay of macro and micro dynamics inherent in the context of micro interactions. Formulating theories in terms of equilibrating dynamics will only succeed empirically if the context of the dynamic process itself is not perturbed. One way of expressing the sociologist's reserve about 'equilibrium' is precisely that such perturbations are, as a consequence of innovations of one sort or another, endemic. So interactive micro processes may, at best, only be conceived as temporarily approaching a steady macro state, before lurching off towards yet another one. Such processes will not give any impression of equilibration unless a heroically impossible level of empirical detail is available ${ }^{20}$, though random variations at the micro level may have the effect of selecting a particular equilibrium in multiple equilibria contexts and local interactions. Certainly as $n$ increases it may prove empirically impossible to check the micro dynamics. There are perhaps two possible reactions to this situation. First, the micro level may be discarded altogether; that is, analysis is then confined to level 4 with a possible commitment to an ontology of system level entities ${ }^{21}$ and a macro equilibrium. Secondly,

17. I shall use the term equilibrium to mean a steady state in macro dynamics (Figure 1).

18. In this respect some summary characteristics of a micro distribution are used to describe the macro state (Figure 1). Sometimes, but seldom, the distribution is conceived as a stochastic process (e.g. a finite Markov chain) which may of course have a dynamic equilibrium. Because social theories are rarely rigorously formulated, whether or not the structural distribution is at a dynamic equilibrium is rarely thought through. Some social theorists use the term equilibrium but unfortunately rarely in a coherent fashion.

19. This is an aspect of supervenience which would dictate that the macro is supervenient on the micro if and only if the macro does not distinguish any state that cannot be distinguished at the micro level. It is generally held that macroscopic properties do not supervene upon the microscopic in the physical sciences. The situation in the social sciences is, however, more debatable; see Abell (1996b). If supervenience is absent, then the map between micro and macro dynamics is many to many.

20. In the extreme, of course, only of the 'history' of the system can be described.

21. That is, to a Durkheimian or structuralist approach. It is sometimes suggested that this approach involves emergent concepts (and equilibria) in the sense that such concepts have no corresponding concepts at the micro level. 
rather brutal simplifications can be attempted at the micro level. Prominent amongst these are assumptions of homogeneous individuals, and a suppression of any interaction (parametric or strategic) between individuals leading to an 'independent individual approximation' (Auyang, 1998).

So let me now draw together the lessons of this introductory section. If we aspire to a unified social science, and by this I mean to explain a system (macro) level state, then:

Which model(s) of the individual should we adopt (if indeed any at all)? What models of interaction should we adopt?

- What role should steady state (equilibria) take in our deliberations?

- How should we conceive of the relationship between the micro and macro dynamics? Is there any room for emergence?

\section{Models of the individual}

Economists have, of course, almost invariably settled upon the rational choice (or action) model of the individual as foundational to their micro theories. The details of what this implies do vary but this need not detain us here. Broadly speaking, individuals are conceived as taking choices (actions in the sociologists' parlance) which (1) optimize their (2) self-regarding exogenously fixed preferences, given (3) their expectations (or beliefs) about the consequences of the chosen and other possible actions (their opportunities) ${ }^{22}$. Sociologists, on the other hand, have variously rejected each one of these assumptions though they have not yet arrived at a widely accepted alternative. Indeed, those of a Durkheimian (or sometimes called structuralist ${ }^{23}$ ) persuasion dismiss the need for a model of the individual at all. For the moment, however, let us put structuralism to one $\operatorname{side}^{24}$. Is there any way of beginning to reconcile micro sociology and economics?

Sociologists have, more or less explicitly (Turner, 1996), proposed a wide variety of models of the individual, many deriving from the phenomenological tradition, which are far too detailed to stand as serious candidates, particularly when $n$ is large and a social explanation (as I have defined it) is sought. It is important, however, not to misinterpret this remark. These models are not necessarily in any sense wrong; rather they cannot reasonably be expected to enter into systematic explanations of the social, when $n$ is large. All we can ask is that our models are not inconsistent with them. The same applies, of course, to detailed psychological models. Cutting away from this diversity, two related contenders appear prominent. First, what I shall call the interactive,

22. This usually leads to a model which maximizes expected utility. Prospect theory is, of course, a development (Kahneman and Tversky, 1979). It is also important that preferences and opportunities are formed independently.

23. The word structuralist has been given many other imprecise meanings by sociologists.

24. If we interpret structuralism as an exclusive attention to level 4 , then we would need to find a concept of 'social causality' not derivable from micro causality (see below). 
referencing or socialization model' whereby individuals are seen as in one way or another (up to innovation ${ }^{25}$ ) deriving their actions (or decision to act) from those with whom they interact (or perhaps observe interacting with others). Although sociologists have not characteristically drawn the distinction, it is perhaps important, in the light of my earlier remarks, to distinguish in this respect between parametric and strategic interactions (socialization). Secondly, the idea whereby individuals follow exogenous norms (or the normative expectations of others ${ }^{26}$ ). The two contenders can, however, for the purposes of this essay, be brought into contact since it is useful to conceive of norm-learning (i.e. normative socialization) in terms of an interactive dynamic ${ }^{27}$. I shall later propose that if it is the case that optimal choice lies at the centre of micro economics, then equally interactive socialization plays a similar role in sociology. If so, the question then arises as to how they may be brought together within a unified perspective.

Returning to the rational choice perspective, many sociologists opine that people just do not, in fact, make decisions (carry out actions) which follow some or all of the various precepts of rational choice. And, of course, they are often correct; it would take very little effort to spot human activities where it would prove difficult to bring them under the auspices of the perspective. But this is to miss the point. We should judge the claims of the rational choice model as a rigorous and parsimonious conception of the individual which is often (not necessarily always) empirically more successful in predicting social states (top right-hand corner of Figure 1) than others. It should be noted that this is, from the sociologist's standpoint, an empirical claim, not an a priori theoretical or normative one. It is nevertheless perplexing to encounter sociologists claiming on a priori grounds that rational choice can never (rarely) in this respect provide an adequate framework. Surely sociologists, given the relative success of economics, must find overwhelmingly secure grounds for rejecting rational choice, at least as a starting point ${ }^{28}$. Furthermore, since what we might term the strict rational choice model (broadly speaking, maximizing expected utility) may be subjected to a wide range of systematic adjustments whilst staying within the broad ambit of the theory (e.g. embracing bounded rationality, relative utility, time-inconsistent preferences, other-regarding sentiments, subjective optimization, various cognitive biase ${ }^{29}$ ), this should further give sociolo-

25. I shall deal with innovation below, but this raises my earlier point about the fleeting nature of a particular equilibrium.

26. Role theory, where people as role holders always follow the normative expectations of other role incumbents, is the most detailed version of this approach. Role theory has, though, had only a marginal impact upon empirical sociology and has been accused of promoting an 'over socialized view of individuals'.

27. I shall try to justify the assertion below.

28. To do so would be to contravene the proposition that people often do the best for themselves in a situation, as they understand it.

29. I am thinking here of, for example, prospect theory. The various biases supported by this theory may, of course, be interpreted as (sometimes) invalidating expected utility theory. 
gists some pause in their eagerness to find another starting point. If one is prepared to permit this rather broad interpretation of the rational choice model, then it is imperative to ask what amongst the 'strict' precepts one must ultimately hang on to whilst still remaining within the jurisdiction of the model. I think it is the idea of optimization, for it is this idea that enables the analyst to make predictions about which actions will be 'chosen' whatever are the preferences or partial understanding or cognitive biases which encumber individuals. If sociologists can find a leading idea which can rival, shall we call it, 'subjective optimization', then an intelligible debate might become possible.

The inclusiveness inherent in my catholic interpretation of rational choice might, whilst allaying the fears of some sociologists and psychologists, nevertheless offend economists. Rightly, the latter often opine; if we are willing to assume in a post hoc manner any sort of preferences or distortions in reasoning, then our theories become well nigh irrefutable. Furthermore, they continue, in what sense is the behaviour/action studied still deemed to be rational?

I adopt what is, I suspect, a rather heterodox and pragmatic view of these matters. Where the boundary should be drawn between what is and is not rational is, I think, of no great moment. The important point is that a very simple model of actions - expected utility theory- provides not only a first explanatory resort, but also a rigorous framework in which the modifications I mentioned above can be progressively introduced. Could we imagine prospect theory (Kahneman and Tversky, 1979) in the absence of expected utility theory? Ultimately a point arrives when it may no longer be helpful to think in terms of modifications of the latter - but only ultimately (see, for instance, a wonderful review paper by Rabin, 1998). Certainly, the research agenda, which embraces the incorporation of psychological insights into rational choice theory, demonstrates a rigour that, by contrast, makes much micro sociology look rather insubstantial. The concern about post hoc irrefutability must of course always provide cause for concern. But equally worrying are economists' attempts to furnish often highly ingenious standard rational choice interpretations of events when an empirically available alternative interpretation, which requires some adjustment of the standard theory, is at hand. I think the answer here, whilst it will not be palatable to many economists, is for us to learn something from sociology and to draw morereadily upon empirically derived testimony of actors (Manski, 1995, Chapter 5). For instance, the repeatedly observed willingness of players to punish in one-shot ultimatum games can, if one wishes, be derived from strict rational choice precepts and an assumption whereby the players think they will in fact be involved in repeated encounters (Gale et al., 1995). However, a little testimony would, no doubt, often reveal a sense of justice and retribution to be involved.

However, I think it more sensible to see them as extending and developing the theory. Similarly also, for instance, Gilboa and Schmeidler's (2001) theory of Case-Based Decisions is conceived as extending rational choice to situations where expected utility does not work well. 
One apparent appeal in adopting a catholic interpretation of rational choice is that it provides space for dynamic models in which biases and incomplete information may be progressively eliminated in the course of individual or social learning. This brings us close to sociology once again, for it can imply learning through interaction or socialization. We should nonetheless be cautious about the universal directionality of 'learning'. Kahneman and Tversky (1979) amongst others point to 'errors of application', which show that individuals who are in possession of appropriate rational principles may not apply them in repeated particular cases. Furthermore, even experts may be overconfident in unpredictable situations.

But how does rational choice theory relate to the sociologists' emphasis upon interactively learned 'normative action'? Empirically one can scarcely doubt that individuals often appear to follow rules or the normative expectations of others rather than to consciously deliberate along the lines required by the precepts of rational choice. The most influential sociological model of 'normative behaviour' — role theory - usually takes the normative expectations which one role holder has of another as exogenous. Thus, social life is largely a matter of learning (socialization) and then abiding by the appropriate rules ${ }^{30}$. Again the picture is often proffered by sociologists as a theoretical insight, but, surely, it is rather an empirical one. Do people sometimes, often, always behave so (i.e.act)? The answer, I suspect, is perhaps frequently, but certainly sometimes. Economists and those sociologists who choose to adopt a rational choice perspective would, however, when faced with empirical evidence of normative actions, seek to endogenize either or both the generation and diffusion of the norms. These objectives would these days also usually be approached from an evolutionary rational choice (often game theory) standpoint. Clearly an interesting question is whether or not norm following is, indeed, optimal and can be constructed as an equilibrium (Elster, 1989). These questions are not normally ones which sociologists ask within the framework of role theory. But there is clearly room here for a unified approach. It is partially a matter of what should be taken as exogenous. Role theorists put the norms (normative expectations) in this position, whilst economists in effect take self-regarding preferences and expectations (i.e. beliefs) about outcomes and constraints as exogenous ${ }^{31}$ and go on to ask how norms could have evolved. However, evolutionary arguments merely shift the sociologists' objection about rational choice to an earlier date that cannot be directly empirically tested other than by the actions themselves, which are licensed by the norms. Certainly, in my view, there is now ample evidence from both evolutionary biology and

30. Defying the norms gives the starting point for an understanding of 'deviant behaviour'.

31. They often do this in the context of an abstract evolutionary test of the optimality of the norms. Furthermore, economists will deploy much ingenuity in order to preserve the assumption of rational choice, particularly self-interest. So, for instance, altruistic norms will characteristically be derived from provident self-interest. It is here that sociologists will often demur, also some economists (Fehr and Gachter, 2000). 
psychology to call into question the all time, all places intrinsic self-interested nature of personkind (Rabin, 1998). Furthermore, norms may evolve in a path-dependent manner from prior normatively constrained situations (Elster, 1989; Abell, 1996a). Be this as it may, there is ample room for an open and constructive debate between economists and sociologists, but this will only be achieved if theoretical sociologists begin to acquaint themselves with the technical rigour of evolutionary dynamics and game theory (see below). Although treating norms as optimally evolved and diffused brings into relief the appropriateness of assumptions about self-regarding rational choice, it can also centre our attention upon the mechanisms of social interaction and socialization whereby norms are learned and transmitted in populations. It seems to me it is here that the ideas which may underpin a unified social science may be sought - by promoting an understanding of when and how socialization takes place in the context of social interaction. Indeed, it may well prove useful to sway somewhat in the direction of sociology by interpreting rational choices (or even bounded rational choices) as embodying particular sorts of norms (rational norms!). It could be that interactive socialization then plays a part in establishing metanorms (normative expectations) indicating situations where either rational calculation or some other sort of normative compliance is what is expected of one (Abell, 1996a). In an evolutionary framework rational choice may still, however, triumph as its precepts may show how the 'situations' have evolved. I believe, however, Elster (1989) is substantially right when he doubts that all social norms can be deemed as optimally evolved. Certainly it is uncontroversial that some norms endure beyond the time when the their rational grounding is pertinent (e.g. shaking hands as a sign that one is not about to draw one's sword). But, furthermore, selection processes in uncertain environments will not necessarily follow an optimizing path. Although it then follows that 'rational choice' theory will, in some way, fail, the very failure prompts a search for an explanation of an anomaly which is conceived within the framework of the theory. In this sense rational choice possesses some paradigmatic privilege (Abell,1996b).

Before moving on, I should like here to insert a personal conjecture. Although I have in this section of the paper argued for an intellectual accommodation between the rational choice and interactive socialized models of the individual, my own view is that we will, in addition, have to find some room for personkind as responsive to ideas of justice. Although this troublesome word can mean many things in different institutional contexts, I am continually impressed by how both altruistic and malign intentions can arise in the context of ubiquitous feelings about justice or fairness, in social interactions (particularly reciprocity). I doubt we can understand much of the social world without taking this into account (and not just as enlightened self-interest in repeated interactions). Fortunately there is some indication that at least some economists may partially agree (Rabin, 1993; Fehr and Gachter, 2000; though, for orthodoxy, see Gale et al., 1995). 


\section{Social interaction}

Most sociologists believe that an understanding of the causes and consequences of social interaction lies at the heart of their discipline; though, if we are to follow my earlier injunction, such understanding is not to be pursued per se but only in so far as some macro state can, in turn, be explained. The approach we may adopt in modelling social interactions is significantly governed by three factors: first, the number of interactants and interactions (i.e. at level 2 in Figure 1); secondly, whether the interactions are conceived as parametric or strategic; and thirdly, whether the consequences are of a significant (i.e. motivating) magnitude to those involved.

Whereas a model of the individual that puts optimal choices at the centre of things is the guiding micro principle for most economists, the parallel principle for sociologists is one which promotes interactive socialization to a similar position. To put it succinctly, people derive their courses of action not from deliberation but from their experience of what others have done in the past (or are currently doing). At least, for many sociologists, this would provide a 'baseline' model which may, nevertheless, be tampered with in various ways (see below) but which would often be subject to systematic or random 'innovation' of one sort or another (i.e. novel actions or unpredictable shifts in action). At a more disaggregate level, sociologists see individual (social) actions as driven by beliefs, affects and values (norms), each of which may be derived in one way or another from those with whom they, often for exogenous reasons, interact. Accordingly sociologists may want to think in terms of modelling those mechanisms (i.e. types of interaction) which underpin cognitive, affective and valuative socialization. This picture, needless to say, also covers normatively driven actions. Just, however, as economists need a rather simple model of the individual (especially when $n$ is large), sociologists also need simple models of interactive socialization. I am not certain what these should be, though I shall make some proposals below.

The challenge underlying the search for a unified social science is one of finding an intellectual accommodation between the relaxed rational choice and interactive models. One apparent way of universally achieving this needs, however, to be discarded at the outset. Economists might naturally see interactive socialization merely as a recipe for endogenizing those preferences, expectations and opportunities which are the ingredients of a standard rational choice interpretation $^{32}$. This is, of course, a possible and attractive option open to exploration as sometimes true. But I think a rather more elaborate picture is needed if sociologists are to be brought on board. A second apparently attractive route also needs, from a sociologist's standpoint, to be approached rather cautiously. A hard line rational choice theorist would wish to endogenize the

32. Note that sociologists speak of beliefs, values and affects, whereas economists use the terms expectations, preferences and opportunities. They can, I think, be transposed. I shall use the economists' terms (see Elster, 1989) as economic theory is so much better developed and it is, thus, sociologists' responsibility to adapt. 
structure of interaction, itself, in terms of rational choice precepts (i.e. the structure would be chosen under the auspices of rational choice theory); namely as the result of optimal search. Most sociologists will resist this possibility, either as a universal or even initial analytical prescription. Their insight is that many actions (or their constituent beliefs, affects and values) are derived from interactions with others who are not chosen in any conscious sense (for the purpose at hand). For this reason the structure of interaction (i.e. with whom one interacts) is usually postulated as exogenous (Coleman, 1990). Clearly, this cannot be a universal analytical prescription either. It is, of course, also feasible that we derive from our interactions guidance as to when and when not to choose rationally and, thus, when and when not to derive things from others. Furthermore, this derivation may also sometimes be the rational thing to do, particularly if deliberation is costly and others are deemed better informed (Conlisk, 1980)! So, where does this leave us? It is not, I believe, entirely clear, but what is evident is that it may be worth asking, in the absence of our ability to endogenize everything pertinent to an understanding of human action, what, when and where should be taken as exogenous.

Although the idea of social interaction is put at centre stage by most sociologists, the discipline has not developed widely accepted ways of understanding the mechanisms involved. Clearly, social interactions may appear extremely complicated and certain theoretical tendencies have consequently driven in the direction of descriptive exhaustiveness ${ }^{33}$. But what is required, particularly when the number of interactants and interactions is large, is a rather simple and robust model. The interactive mechanisms which can (partially) determine the actions of individuals (level 2 in Figure 1) will be as varied as are the descriptions of the 'conditions of action'. In this respect, as we have noted, economists usually deploy the vocabulary of exogenous preferences, expectations and opportunities, whereas sociologists speak of values, beliefs, affects and norms. Actions can, in principle, be shaped by interactions impacting upon any one or a number of these descriptors.

I should like to contrast three models of interaction as follows:

(i) The endogenous structure model.

(ii) The endogenous interaction exogenous structure model (structural effects).

(iii) The exogenous interaction exogenous structure model (contextual effects).

The endogenous structure model (type i) is closest to the classical rational choice perspective. Individuals exogenously endowed/placed with 'conditions of action' (Figure 1) (e.g. preferences, beliefs and opportunities) generate a structure of interaction (with other individuals) in search of 'information' which may in turn modify their conditions and, thus, their prior actions. This is the approach with which sociologists appear not to be very happy as they are inclined to see most situations as ones where there is a received (exoge-

33. As $n$ dwindles, the complex models may become more appropriate. 
nous) 'structural history'. In practice, I suspect, we shall have to begin to think in terms of the co-evolution of structures and the actions of individuals. Meanwhile, we may look to Jackson and Wolinsky (1998) for the most promising approach, from a sociologist's standpoint, to endogenizing structure. Starting from a rational choice perspective, these authors search for equilibrium structures where both direct and indirect links bring benefits (e.g. information) but where actors only bear the costs of direct links. In this set-up indirect links can provide a positive externality for those who make use of them but, nevertheless, do not sustain the cost of their maintenance. It is really the optimality assumption of the rational choice perspective which does the hard work here. One could easily modify the model, by introducing altruistic and/or malign objectives or, indeed, any others. This observation underscores my earlier remark about the flexibility of rational choice theory. I have argued for a number of years now (1996b) that a judicious combination of network and rational choice ideas can provide the framework for the construction of genuine social theory.

Sociologists are, however, inclined to make their opening gambit with a model which makes the structure of interaction exogenous (often constructed for 'other reasons') ${ }^{34}$. Then the simplest model (type ii) is one where the focal actors/agents merely derive (perhaps in an iterative manner) their actions (or their observable outcomes) from those actors to whom they are (exogenously) connected (Doreian, 1981; Friedkin, 1990). This leads (at the micro level 2 in Figure 1) to patterns of endogenous interaction (structural effects) within the framework of an exogenous structure. Sociologists, however, often in addition postulate (type iii) patterns of exogenous interaction, i.e. what they often term 'contextual effects'.

As Manski (1995, Chapter 7) has observed, there appears to be a marked tendency for sociologists to theorize in terms of endogenous interaction but then to empirically test contextual models. Since both mechanisms are concurrently possible, it is important to clearly separate them, both theoretically and empirically, and also to differentiate them from any 'correlation effects' 35 .

An outline example may make these ideas rather more accessible. Assume we are interested in how the actions of individuals helping others (bottom right-hand corner of Figure 1) contribute to 'group cohesion' (top right-hand corner). Group cohesion may be conceptualized as the mean level of helping within a group (arrow 3 is then one of aggregation). Our intellectual concern,

34. Such an analytical starting point seems quite consistent with the precepts of institutionalism in economics.

35. See Manski (1995). Correlation effects arise when individuals are grouped and the grouping variable(s) correlates with the endogenous variable. Identification problems arise in separating the impact of endogenous and exogenous interactions and correlation effects. Lagged models help to sort out these problems but this directs attention to an understanding of the dynamics of interaction effects. This could be an active area of research in any attempt to generate a unified social science. 
thus, starts at the 'social' level and initially tracks back to the micro level. We now pose the question about how the 'conditions of helping actions' (type 2 mechanism in Figure 1) generate the distribution of actual helping of others within groups. An endogenous interaction and exogenous structure mechanism (ii above) would work from assumptions that:

(a) a 'historical' structure of 'who interacts with whom' is given (imposed!) (exogenous);

(b) helping actions (the endogenous variable) are shaped (stochastically) by: - a vector of exogenous personal variables (e.g. gender, age, 'perception of outside group opportunities', etc.)

- the auto-correlation of the endogenous variable (i.e. helping), in virtue of (a), which determines the pattern of social interaction.

In a large $n$ situation the details of the exogenous structure may be practically unobservable and replaced by, say, the mean level of help within the group (sociologists call these 'structural effects'). If this is done, of course, it is better to think in terms of a dynamic (lagged) model since 'help' appears on both sides of the estimation equation (see footnote 35).

If we add to this picture (which you may think is already complicated enough) the possibility of exogenous auto-correlation (contexted effects) whereby the (exogenous) structure (a) also, for instance, introduces inter-individual patterns of interaction determining the 'perception of outside group opportunities', then we are in the realm of model (iii) above. Relaxing assumption (a) then leads to the additional complication that the structure of who interacts with whom is itself not given. These are the sorts of issues that serious social theory is going to have to address if common ground with economists is to be found. Since I wrote the first draft of this paper I find a start has been made (Durlauf and Young, 2001).

\section{Combining rational choice and interaction}

If we may conceive of a unified social science wherein a relaxed conception of individual rationality is carefully combined with dynamic notions of socialization through interaction, then what is the theoretical way forward? Sociologists have developed a number of dynamic models where the interaction is by and large parametric, usually depending upon simple density dependence contact or copying ${ }^{36}$ (Coleman, 1964). These more or less complex deterministic and stochastic diffusion models have, however, not entered mainstream social theory to any appreciable extent (though see Fararo, 1989). Furthermore, over the years a number of empirical studies have made use of con-

36. Coleman introduced diffusion models where estimates of the rate constants can be derived from the (exogenous) structure of interaction. 
textual and endogenous interaction models where the conditional expected value of the appropriate variable is used to proxy the details of micro interactions (Hauser, 1970; Jenks and Mayer, 1989; Crane, 1991; Mayer, 1991). But again, even though to ignore such effects, if they exist, will bias the estimated impact of any exogenous variables, empirical sociologists still almost invariably assume micro unit independence ${ }^{37}$. Few systematic attempts have been made, at the theoretical level, to explicitly model interactions. This, however, is the direction we must go in to return to the roots of the discipline.

In doing so it is probable that the analysis of strategic rather than parametric interactions will gain in prominence (Abell, 2001). This being the case, it is in the direction of some adaptation of evolutionary game theory that we might look for a unifying theoretical inspiration. One attraction of doing so, from the sociologists' standpoint, is that evolutionary game theory has no need of strong rationality assumptions. Rather, dynamic interactive processes are usually deemed to myopically procure rationality over a period of time. Thus, equilibrium is not achieved as a consequence of rational actions, but actors acquire rationality if and when an equilibrium is achieved. With myopic adjustment, because actors do not take account of how their own actions affect others, they behave as though the world is stationary even though it is not. But this is a useful approximation. Furthermore, the equilibrium can be disturbed in a predictable manner by the entry of new actions/strategies (i.e. equilibrium disturbing innovations, as noted above, are often urged by sociologists). These conceptions of equilibrium and rationality, thus, seem rather compatible with many traditional sociological concerns. Evolutionary models work well when $n$ is large (or alternatively, the number of interactions is large), the strategic situations modelled are not overcomplicated and the ratio of pay-off returns to learning and switching costs (i.e. changing strategy) are of sufficient magnitude to motivate actors. Much of the early work on evolutionary models has assumed patterns of random pairwise interactions between actors ${ }^{38}$. Sociologists will wish, however, to complicate this picture in several respects. First, as we saw earlier, they will often start with an exogenous structure of interaction (i.e. a picture of who interacts with whom). In large social systems this structure will characteristically comprise a loosely linked aggregate of more densely interacting local systems. We have little knowledge of how to model and sample from such systems (Marsden, 1990), though it is significant that it is not sociologists but economists who have made a start ${ }^{39}$. Furthermore,sociologists will also want to

37. We need to control for exogenous and endogenous interaction effects in order to ascertain the independent effect of any exogenous variables. This observation tends to suggest that the routine use of regression-based models (without allowances for interactions) is not appropriate.

38. Simple diffusion models also have this characteristic.

39. I refer here to stochastic stability with local interaction, e.g. Blume $(1993,1995)$ and Ellison $(1993,1995)$. 
model the structure itself as evolving, in the sense that current interactions will enhance (weaken) the probability of future use (i.e. learning by using a relationship). So, we will need to model the dynamics of the structure of interaction itself. We will, thus, begin to think in terms of the co-evolution of the structure of interaction and the actions (strategies) of the actors.

Secondly, sociologists will allow for a (possibly evolving) 'structure of observability', whereby actors are able to observe and react to the interaction of others (i.e. a picture of who observes whom interacting). Thirdly, as a consequence, actors will acquire reputations (for acting in certain ways) which will change through time. Thus, to put it succinctly, much more local detail in the patterns of interaction and reference will be needed than is provided in most current evolutionary models for them to prove convincing to sociologists. It is, of course, in light of my earlier remarks concerning simple models of interaction, important to find some happy medium. It will not prove feasible for instance to impose the detailed models sometimes used in small group research when $n$ grows [e.g. the full structural models as in Friedkin (1990)]. Some progress has been made by adopting 'neighbourhood effects' into the evolutionary dynamics of conventions derivative of simple coordination games (Ellison, 1993). Multiple conventions (i.e. differing equilibria) are possible which may even be non-exclusive (Sugden, 1995; Goyal and Janssen, 1997). Although the diffusion models developed by sociologists have, by and large, been parametric in inspiration, it may prove instructive to picture the spread of 'traits' (e.g. beliefs, values, etc.) as essentially strategic (in the sense of finding a coordination equilibrium).

The leading idea in evolutionary dynamics is that actors tend to switch, according to some dynamics specification, from less successful to more successful strategies (actions). The reference group for relative success can vary with either or both of the structures of interaction or observability (which is often taken to be the population). Three measures of success traditionally enter the dynamic specification. First, the current success of a particular strategy when interacting with other strategies; secondly, some measure of the success of other strategies (e.g. their mean success or the most successful); thirdly, the proportion (i.e. density) of the actors adopting the particular strategy. Density-dependent dynamics will capture the historic success of a particular strategy, whereas relative success dynamics concentrate upon the current situation. It will prove useful to conceive a continuum of dynamic models ranging from, at one end, dynamics, which are purely density-dependent, to the other end where, rather, they depend entirely upon success ${ }^{40}$. The standard Taylor-Jonkers (replicator) dynamics can then be proven to lie at the centre point of this continuum, weighting density and success equally (Bendor and Swistak, 1996).

40. Models will characteristically contain terms for both. 
Pure density-dependent dynamics (usually proposed by sociologists) are most apposite where social interactions are what I above termed parametric. The individual propensity to adopt a particular action (strategy) depends upon the (perhaps local) density of contacts (exogenous structure) with others. The question then arises as to how complicated the picture of (parametric) social interaction should be. Simple deterministic and stochastic models of diffusion (usually logistic in form with an upper equilibrium at a carrying capacity) have dominated the field (Coleman, 1964). The model of interaction underlying this dynamic formulation is one of simple pairwise interactions between those with a 'trait' and those without. In the standard model, the rate of propagation of the trait is proportional to the product of the members with and without the trait. This is appropriate where the (exogenous) structure is either complete (i.e. each pair of interactants is equally likely), or incomplete but where the interaction patterns are, nevertheless, random. Surely some unified theoretical and research programme is possible here, which will explore the steady state implications of different dynamic specifications across the density to success continuum matched, where appropriate, with different types of strategic interactions. I suspect there are some rather deep theorems to be discovered in this respect, signalling the sensitivity of steady state equilibria to different dynamic models and the shape of the functions linking transition probabilities with density and relative success.

It is already known that with stochastic dynamics and patterns of local interactions path-independent equilibria are possible (Blume, 1993; Ellison, 1993) within the compass of a reasonable time period ${ }^{41}$. Sociologists have always, in a rather ill-specified way, been inquisitive about when and where history-dependent and -independent predictions hold. The framework within which to think about these matters is now taking shape.

Sociologists will want to push things a little further by introducing status (or reputation) and threshold effects into their dynamic picture. It is perhaps useful to contrast the sociologists' and economists' treatment of status/reputation. By and large economists have not tampered with standard assumptions about arguments in utility functions when conceiving of reputation effects. Rather, the acquisition of a reputation is almost invariably conceived as instrumental to the achievement of standard utilities (usually in a repeated game context, i.e. repeated interaction). Thus, it is in the rational self-regard of actors to seek reputations. This is clearly the most parsimonious way for economists to incorporate 'status-seeking' into their theories (Nowak and Sigmund,1998).

Sociologists (Coleman,1990), on the other hand, in so far as they have addressed the issues systematically, have often chosen to picture status as an

41. One may wish to think in terms of a larger continuum ranging from historical density dependence (i.e. lagged measures of density) to forward-looking success measures (e.g. rational expectations). The continuum mentioned in the text is, in effect, embedded in this larger continuum. 
independent objective ${ }^{42}$. If so, dynamic specifications would not only contain terms for relative success and density, but also status at some point on the density-success continuum. Whilst it seems initially sensible to follow the economists' instrumental view-point, it may be that independent status-seeking does, at some point, play a role in dynamics. The significance of this possibility can be appreciated by considering Prisoner's Dilemma type strategic interactions. If the benefit (utility) derived from the status of cooperating is greater than the cost of the cooperation, then the Prisoner's Dilemma is transformed into a coordination game. So, the nature of strategic interaction can be fundamentally transformed. Contrast, for instance, social capital interactions where: (a) helping others leads to a reputation for helping (i.e. status) which, in turn, increases the likelihood of being helped at a later date (i.e. generalized reciprocity), and (b) helping confers status which boosts one's utility. The issue is that, depending upon the assumptions one makes, the dynamics will vary dramatically. Furthermore, it does appear that independent status may well acquire the characteristics of a relative utility measure. This being the case, the status of others may exert a negative externality upon oneself (i.e. status is derived from the difference between my reputation and yours and vice versa). Here dynamics may lead to a status race without any increase in mean satisfaction (utility). For example, happiness per capita does not seem to increase with wealth per capita above a certain threshold. This may be an example of emergent status effects (see next section).

Finally, sociologists (Granovetter, 1978, 1982) seem to believe that threshold effects are endemic in dynamic processes. So, for instance, in a given population there will be a distribution of the probability of changing strategy (action) depending upon the (often local) density of the strategy in the population. Some will move easily, others with more difficulty. This may equally apply to relative success.

The way of reasoning embodied in the preceding paragraphs depends upon a large $n$ and/or number of interactions. An important question is how we might proceed if these numbers are not high (Abell, 2001) when narrative models are, I believe, relevant (Abell, 1988). I shall, however, leave these matters to another occasion.

\section{A note on the macro level (level 4) -emergence?}

As noted earlier, sociologists often appear to seek formulations of causal connections which operate exclusively at the macro level (level 4 in Figure 1). This approach to matters I have variously described as the Durkheimian or structuralist programme. Economists are less prone to this sort of endeavour, rather,

42. Stochastic dynamics without local interactions have a low probability of settling upon the (risk dominant) equilibrium in coordination problems in a reasonable period of time. Thus, in the face of multiple equilibria (e.g. coordination) the equilibria arrived at will be pathdependent. 
almost invariably, wishing to provide 'micro foundations' for any macro connections. By this they usually mean that, in some way, connections between macro states can be aggregated or deduced from assumptions (or perhaps observations) of connections between micro states. So in the simplest possible interpretation, a specified functional (dynamic) relationship at level 2 (where measures pertain to individuals) could be aggregated so that the individual level function form could be replaced by one in terms of, say, mean values ${ }^{43}$. Although some empirically orientated sociologists (e.g. Hauser, 1970) have taken a similar stance, leading to literatures on ecological correlation and contextual effects, there is no doubt that the issue of 'reduction' (or sometimes called methodological individualism) does appear to significantly divide the disciplines. In its most extreme manifestation we encounter many sociologists claiming that (macro) sociological causal connections are in some sense irreducible or emergent.

Emergence has two aspects: first, an assertion that macro concepts (states) cannot be connected to micro concepts; secondly that the macro social causal connection itself cannot be reduced to causal links 1, 2 and 3 in Figure 1. Although the concept of emergence has acquired a number of meanings, I should like to distinguish between strong and weak versions. By strong emergence I shall mean the doctrine that in principle macro states cannot be reduced to (or deduced from) micro states; nor can macro causal connections. Weak emergence, on the other hand, may be used to describe situations where, in practice, given our present intellectual understanding, it proves impossible to find an acceptable micro reduction.

Related to strong emergence are three further ideas. First, the map from macro states to micro may be many to one (the many to one map the other way round is not controversial). Secondly, the idea that macro states cannot be obtained by simply averaging or aggregating over micro states, particularly in respect of inference 3 in Figure 1. Thirdly, if the micro dynamics at level 2 in Figure 1 turn out to be non-linear, then it is conceivable that chaotic regimes will emerge. If so, they may be described as emergent since they are clearly not supervenient. Whether chaotic regimes will play a significant role in the future of social science is, I think, at the moment not clear. There is, of course, a lot of loose talk, but that is all. Nevertheless, if as I have argued evolutionary dynamics come to hold a central role, then 'edge of chaos' arguments may come to prominence, as will networks 'between order and randomness' (Watts, 1999).

I may not be well informed, but I suspect economists show little sympathy with strong emergence. It is relatively easy to see why sociologists tend to differ and speak of emergence. It is because they start with the idea of interaction and thus are held, at the micro level, to modelling complex patterns of interaction, whereas economists are more likely to adopt an exogenously endowed

43. Status may also be best conceived as relative. 


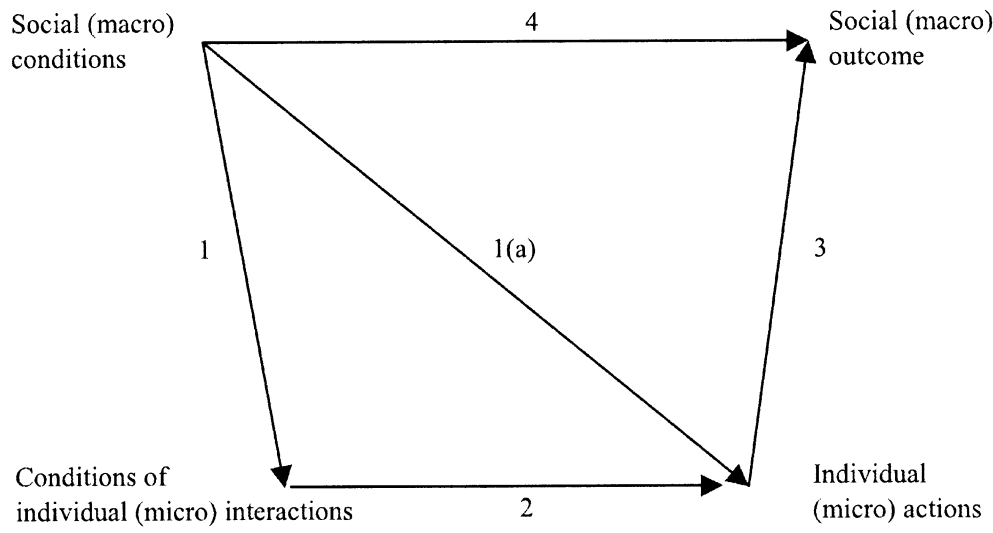

Figure 2.

independent model of the individual (but see Durlauf and Young, 2001). They need also, in order to render this approach cogent, to assume that individuals' opportunities (feasibility set) and preferences are independent of each other. So, even if economists suspect inter-individual interactions at level 2 in Figure 1, they will, by and large, ignore them; to put it succinctly, they adopt an independent individual approximation. This has the additional advantage that the micro-macro connection (number 3 in Figure 1) can be interpreted as a mean value. Sociologists face more difficulties in effecting reduction. But let us first dispose of strongly emergent causality. Such would amount to the idea that there is some form of 'macro' causality which is logically independent of micro actions. I can attach no meaning to such an idea; of course, we may not be able to formulate the causal structure implied by 1, 2 and 3 (Figure 1), but this is a matter of weak, not strong, emergence.

Sociologists inspired, as we have had occasion to note, by Durkheim (and structuralists) are inclined to speak of emergent 'social forces' acting at the top left-hand corner of Figure 1 impacting the (social) outcome at the top righthand corner. But, if we can attach no clear meaning to the idea of 'social causality' (at level 4) operating independently of individual (micro) actions, then what is really being claimed (Abell, 2001) is that any causality in fact runs from the top left-hand corner to the bottom right-hand corner (as in Figure 2$)^{44}$. That is to say, causality operates independently of the structure of interaction (context of individual action in Figure 1). Such causality can itself invite two interpretations: first, non-emergent, whence mechanism 1(a) is merely the transitive causal closure of the mechanisms working through 1 and 2. Note

44. Note this formulation in effect revises arrow 1 in Figure 1. The conditions under which such aggregations can be effected are well understood. 
here, also, that mechanism 4 is now the transitive closure of 1 (a) and 3 . Secondly, a causal picture whereby 1(a) operates independently of 1 and 2 either because the latter do not operate, or because they do operate alongside mechanism 1(a). Certain diffusion models proposed by sociologists, for instance, take these various forms where the rate of propagation can, variably, depend upon pairwise interactions and the absolute number of non-converted individuals. The endogenous and exogenous interaction models mentioned above, which proxy interactions by conditional expectations varying across reference 'groups', are non-emergent variants of this theme. They are, indeed, independent individual approximations which are designed to suppress the minor details of interactions in terms of a mean field effect. They, in practice, treat each micro individual as reacting independently of others by an (intra-group) identical force (i.e. the appropriate conditional expectation).

We are now finally in a position to define a working model of weak emergence. Relation 1(a) is weakly emergent if there exists no practical way of deriving it from an independent individual approximation. Thus, 1(a) may be (weakly) emergent but 4 is the transitive closure of 1 (a) and 3 and can only be derivatively emergent if 1 (a) happens to be so.

Returning to the theme of co-evolution: in general both social forces 1(a) and interactive mechanisms (2) may determine actions and,thus,social actions. But the mix can vary over time. At one extreme we have a 'community' where interactive mechanisms solely determine the actions and at the other extreme social forces (i.e. weak emergences) play this role. Note that when the latter is the case the endogenous and contextual interaction model reduces to the impact of correlation effects only. ${ }^{45}$

Thinking in these terms should, I trust, enable economists and sociologists to unite with a common perspective.

\section{Conclusions}

It is difficult to draw conclusions from a paper like the foregoing. So, rather than try, let me anticipate one or two possible objections. First, why place such a heavy emphasis upon social interaction (as the conditions of action)? I suppose some will not agree that it is the sociologist's leading idea; in particular it may be asked what has happened to 'culture'. Surely sociology is the 'science' which promotes culture (however we define it) as the major shaping force of our actions. There is, in fact, no inconsistency with this proposal and the ideas I have promoted. Indeed, 'culture' may, for certain purposes, be taken as exogenous, residing at the top left-hand corner of Figure 2, which then

45. Let $y$ be the outcome, $g$ a grouping variable and $x$ an exogenous variable. Then:

$$
E(y / g, x)=\alpha+\beta_{1} E(y / g)+\beta_{2}(x / g)+\beta_{3} g+\beta_{4} x+\mu
$$

So when correlation effects solely operate, $\beta_{1}=\beta_{2}=\beta_{3}=0$. 
impacts our actions either via mechanisms 1 and 2 (i.e. through interaction), or 1(a) (i.e. as a 'social force'). It, thus, by derivation fashions that 'social outcome' through 4 (i.e. non-emergent). One notable way in which this could be operative is through 'focal point' selection of equilibria at level 2 (Schelling, 1978).

Finally, some sociologists will promote various conceptions of 'power' as a leading idea. Once again battle lines should not be drawn. Interactions involving both inter-personal and inter-group (class or whatever) power may well be amongst the most important to study in determining actions and group outcomes.

\section{References}

ABeLl, P. (1988). The Syntax of Social Life. Oxford: Oxford University Press.

- (1996a). «Homo sociologicus: Do We Need Him/Her?». In: Turner, S.P. (ed.). Social Theory and Sociology. Cambridge, MA.: Blackwell, p. 229-34.

- (1996b). "Sociological Theory and Rational Choice Theory». In: TuRner, B.S. (ed.). The Blackwell Companion to Social Theory. Oxford: Blackwell, p. 252-73.

- (2001). "Causality and Low Frequency Complex Events». Sociological Methods and Research, 30, 57-80.

Abell, P.; Reyniers, D. (2000). "Generalised Reciprocity and Reputation in The Theory of Co-operation: a Framework». Analyse und Kritik, 22,3-18.

Arrow, K.J. (1983). Collected Papers of Kenneth J. Arrow (Vol. 2), General Equilibrium. Cambridge, MA.: Harvard University Press.

AuYANG, S.Y. (1998). Foundations of Complex-System Theories in Economics, Evolutionary Biology and Statistical Physics. Cambridge: Cambridge University Press.

BENDOR, J.; SWISTAK, P. (1996). «The Controversy about the Evolution of Co-operation and the Evolutionary Roots of Social Institutions». In: GASPARSKI, W.; Mlicki, M.; BanTHAY, B. (eds.). Social Agency. New Brunswick, NJ.: Transaction Publishers.

Blume, L. (1993). «The Statistical Mechanics of Strategic Interaction». Games and Economic Behaviour, 5, 387-424.

- (1995). «The Statistical Mechanics of Best Response Strategy Revision». Games and Economic Behaviour, 11, 111-45.

Coleman, J.S. (1964). Introduction to Mathematical Sociology. Glencoe: Free Press.

- (1990). Foundations of Social Theory. Harvard: Belknap.

ConLISK, J. (1980). «Costly Optimisers Versus Cheap Imitations». Journal of Economic Behaviour and Organization, 1, 275-93.

CrANE, J. (1991). "The Epidemic Theory of Ghettos and Neighbourhood Effects on Dropping Out and Teenage Childbearing». American Journal of Sociology, 96, 1226-59.

DOREIAN, P. (1981). «Estimating Linear Models with Spatially Distributed Data». Sociological Methodology. Glencoe, IL.: Free Press.

Durlauf, S.N.; Young, H.P. (eds.) (2001). Social Dynamics. Cambridge MA.: MIT.

ELLISON, G. (1993). «Learning Local Interaction and Co-ordination». Econometrica, $61,1047-71$. 
- (1995). «Basins of Attraction and Long Run Equilibria». Mimeo. Cambridge, MA.: MIT Press.

ELSTER, J. (1989). The Cement of Society: a Study of Social Order. Cambridge: Cambridge University Press.

FARARO, T. (1989). The Meaning of General Sociological Theory. Cambridge: Cambridge University Press.

FEHR, E.; GACHTER, S. (2000). «Fairness and Retaliation:the Economics of Reciprocity». Journal of Economic Perspectives, 14, 159-81.

Friedkin, N. (1990). "Social Networks in Structural Equation Models». Social Psychology Quarterly, 53, 316-28.

Gale, J.; Binmore, K.; Samuelson, L. (1995). "Learning to be Imperfect: the Ultimatum Game». Games and Economic Behaviour, 8, 56-90.

Giddens, A. (1989). The Constitution of Society: Outline of a Theory of Structuration. Cambridge, Polity Press.

Gilboa, J.; Schmeidler, D. (2001). A Theory of Case-Based Decisions. Cambridge: Cambridge University Press.

Goyal, S.; Janssen, M.C.W. (1997). «Non Exclusive Conventions and Social Coordination». Journal of Economic Theory, 77, 34-57.

GranovetTer, M. (1978). "Threshold Models of Collective Behaviour». American Journal of Sociology, 83, 1420-43.

- (1982). "The Strength of Weak Ties: a Network Theory Revisited». In: MARSDEN, P.V.; LIN, N.(eds.). Social Structure and Network Analysis. Beverly Hills, CA.: Sage, p. $98-120$.

Hauser, R. (1970). «Context and Consex: a Cautionary Tale». American Journal of Sociology, 75, 645-64.

JaCKSON, M.O.; Wolinsky, A.(1998). «A Strategic Model of Social and Economic Networks». Journal of Economic Theory, 71, 44-74.

Jenks, C.; MAYER, S. (1989). "Growing up in Poor Neighbourhoods:how much does it matter?». Science, 243, 1441-5.

Kahneman, D.; TVERSKY, A. (1979). «Prospect Theory:an Analysis of Decision under Risk». Econometrica, 47, 263-91.

MANSKI, C.F. (1995). Identification Problems in the Social Sciences. Cambridge, MA.: Harvard University Press.

Marsden, P. (1990). «Network Data and Measurement». Annual Review of Sociology, $16,435-63$.

MAYER, S. (1991). «How Much Does a High School's Racial and Socio-economic Mix Affect Graduation and Teenage Fertility Rates?». In JenKS, C.; PETERSEN, P. (eds.). The Urban Underclass. Washington, DC.: Brookings Institution.

NowAK, M.A.; SigmUND, K. (1998). «Evolution of Indirect Reciprocity by Image Score». Nature, 393, 573-7.

Rabin, M. (1993). "Incorporating Fairness into Game Theory and Economics». American Economic Review, 83, 1281-302.

- (1998). "Psychology and Economics». Journal of Economic Literature, 36, 11-46. SCHELling, T. (1978). Micromotives and Macrobehaviour. New York: Norton.

Sugden, R. (1995). "The Co-existence of Conventions». Journal of Economic Behaviour and Organization, 28, 241-56.

Turner, B.S. (1996). Social Theory. Oxford: Blackwell.

WatTs, D.J. (1999). Small Worlds: The Dynamics of Networks Between Order and Randomness. Princeton, NJ.: Princeton University Press. 